\title{
OBTENCIÓN DE UN EXTRACTO ANTOCIÁNICO A PARTIR DEL RESIDUO DE MORA DE CASTILLA (RUBUS GLAUCUS BENTH)
}

OBTAINING AN ANTHOCYANIN EXTRACT FROM ANDEAN BLACKBERRY WASTE (RUBUS GLAUCUS BENTH)

Nubia Liliana Becerra-Ospina*

https://orcid.org/0000-0003-2198-8479

Angie Pamela Muñoz-Betancourt**

https://orcid.org/0000-0002-7385-5048

Andrea Lizeth Lucero-Bustos***

https://orcid.org/0000-0003-4997-1572

UNIVERSIDAD DE AMÉRICA, COLOMBIA

Recibido: 10 de octubre de 2019

Aceptado: 6 de diciembre de 2019

DOI: https://doi.org/10.29097/2011-639X.298

\section{Resumen}

Este artículo estudia la extracción con disolventes de antocianinas a partir del subproducto generado en el despulpado de la mora de castilla, utilizando extracción con etanol en concentración de 75\%, 85\% y 96\% (v/v), mediante método soxhlet y ultrasonido. El mayor rendimiento de extracto se obtuvo en la extracción asistida con ultrasonido con etanol $96 \%$ (v/v), produciendo $65.85 \pm 5.25$ g de extracto por cada 100 g de residuo en concentración de $661.944 \mathrm{mg}$ de cianidin-3-glucosido/L. El extracto producido puede ser utilizado como colorante natural, considerando que se verificó la presencia de antocianinas mediante espectrofotometría y la prueba cualitativa de cambio de color según el pH.

Palabras clave: antocianinas, extracto antociánico, extracción, mora, ultrasonido.

\section{Abstract}

Andean blackberry is one fruit wide industrial interest and recognized for anthocyanins content. The solvents extraction of these components from blackberry byproduct was studied by extraction with ethanol, two method were used, soxhlet and ultrasound. The concentration was varied on $75 \%$, $85 \%$ y $96 \%$ vol. It was identified that the extraction with ultrasound with $96 \%$ vol. ethanol, generated the highest yield of $65.85 \pm 5.25 \mathrm{~g}$ extract/100 g waste with $661.944 \mathrm{mg}$ cyaniding-3-glucoside/L. The extract produced can be used as a natural dye considering that the presence of anthocyanins was verified by spectrophotometry and the qualitative color change test according to the $\mathrm{pH}$.

Keywords: anthocyanins, anthocyanin extract, extraction, blackberry, ultrasound.

\footnotetext{
* M Ingeniera química, especialista en Ingeniería Sanitaria y Ambiental, magíster en Ingeniería Química. Docente asociado del Departamento de Ingeniería Química, Fundación Universidad de América, Bogotá, Colombia. $₫$ nubia.becerra @profesores.uamerica.edu.co

** Ingeniera química, Fundación Universidad de América, Bogotá, Colombia.

*** Ingeniera química, Fundación Universidad de América, Bogotá, Colombia.
} 


\section{INTRODUCCIÓN}

La mora de castilla (Rubus glaucus benth) es una fruta originaria de la zona andina tropical alta de América, con gran variedad de especies ampliamente cultivadas en clima tropical (Ministerio de Agricultura y Desarrollo Rural y Dane 2013). Se cultiva en forma silvestre en muchos países por su follaje (Aramwit, Bang y Srichana, 2010). Los frutos de mora se pueden destinar a consumo en fresco, aunque, por ser perecederos, se prefiere el procesamiento en jugos, pulpas, vinos, entre otros medios (Khalifa, Zhu, Li y Li, 2018).

La mora de castilla es una fruta compuesta por $85 \%$ de agua y materia seca, formada mayoritariamente por carbohidratos, compuestos nitrogenados, minerales, entre otros. La fracción aprovechable en pulpa oscila entre el 62 y el 74\% (Ayala, Valenzuela y Bohórquez, 2013), lo que sustenta su importancia a nivel comercial. Como el procesamiento de frutas genera altos volúmenes de residuos orgánicos que terminan en los rellenos sanitarios y considerando sus limitaciones de capacidad, se hace necesaria la valorización de estos materiales mediante diferentes tecnologías (Yepes, Montoya y Orozco, 2008). Dentro de las opciones de aprovechamiento de los residuos vegetales se encuentran la alimentación animal y humana, la obtención de biogás y la extracción de componentes funcionales o nutricionales. Se han extraído compuestos polifenólicos desde subproductos industriales, como orujo de uva roja, cebolla, aceitunas, tomates y cáscaras de residuos de limón (Panić, Gunjević, Cravotto y Radojčić, 2019).

En el caso particular de la mora, en el proceso de despulpado se genera un subproducto compuesto por las semillas y parte del material comestible; este residuo puede ser aprovechado para la recuperación de las antocianinas como colorantes alimentarios (Guo et al., 2019). El interés por los pigmentos antociánicos se ha incrementado en los últimos años (Garzón, 2008). Según los aportes realizados por Aramwit et ál. (2010), Cai et al. (2016) y Heinonen et al. (2016), las antocianinas son benéficas para la salud por su actividad antioxidante, además de tener propiedades antimicrobianas, antivirales y antiinflamatorias, por lo que la ingesta alimentaria a largo plazo de antocianinas puede reducir el riesgo de cáncer, enfermedades cardiovasculares, entre otras (Heinonen et al., 2016). A pesar de las ventajas de las antocianinas como potenciales sustitutos de los colorantes artificiales, hay aspectos que limitan su aplicación industrial, como son la baja estabilidad y la falta de disponibilidad de material vegetal (Garzón, 2008).

Las antocianinas son compuestos polifenólicos, principales responsables del color rojo, morado, violeta y azul de las frutas, verduras y cereales (Garzón, 2008). Dentro de los principales componentes de las antocianinas se destaca la cianidina-3-O-glucósido, la cianidina-3-O-rutinósido, la cianidina-3-O-(6”-malonil-glucósido) y la cianidina-3O- (6”-Dioxalilglucósido), los dos últimos en menor proporción (Aramwit et al., 2010; Guo et al., 2019). Particularmente, se reporta un contenido de $3.000 \mathrm{mg} . \mathrm{kg}^{-1}$ de peso fresco en las frutas de mora (Cai et al., 2016; Khalifa et ál., 2018).

El estudio de metodologías para la extracción y aplicación de las antocianinas como colorantes naturales genera una alternativa para la producción de colorantes sintéticos, como la tartrazina, la cual abarca un gran rango de productos alimenticios en el mercado 
(Parra, 2004). Dentro de las tecnologías disponibles para la obtención de antocianinas se encuentra la extracción con disolventes polares (Panić et al., 2019). Adicionalmente, se establece que la forma química y estabilidad de las antocianinas dependen del valor del $\mathrm{pH}$, por lo que es una variable de control del proceso. Chandrasekhar, Madhusudhan y Raghavarao (2012) obtuvieron en sus ensayos con diferentes medios de extracción el etanol acuoso acidificado $(50 \%, \mathrm{v} / \mathrm{v})$; en este caso, el mejor resultado fue un extracto con $390.6 \mathrm{mg} / \mathrm{L}$ de antocianinas.

Liu, Yang, Zhou, Wen, \& Dong (2019) investigaron el efecto de la concentración de etanol variando entre 0 y $60 \% \mathrm{v} / \mathrm{v}$; los resultados indicaron que el contenido de antocianinas en el extracto aumentó a medida que aumentó la concentración de etanol; aquí se obtuvieron $31.16 \mathrm{mg}$ de antocianinas totales/100 g de patatas moradas con etanol al 30\% v/v, con mínima cantidad de ácido crítico e irradiación de microondas.

Cai et al. (2016) hacen un comparativo entre diferentes métodos de extracción; en orden decreciente en cuanto a la eficiencia de extracción de antocianinas, los métodos estudiados fueron: extracción acelerada con solvente (ASE), extracción asistida por ultrasonido (UAE) y extracción convencional (CE); con esta última tecnología, el rendimiento fue de 218-244 mg/100 g de peso seco. Según Huang et ál. (2019), la UAE facilita la penetración del disolvente en la matriz sólida. Este efecto se evidencia en el estudio de Corona-Jiménez, Martínez Navarrete, Ruiz-Espinoza y Carranza-Concha (2016); de acuerdo con la investigación, “el proceso asistido por ultrasonido mostró mayor capacidad de extracción respecto que los métodos convencionales” (p. 403); además, indica que el efecto del método depende de la potencia ultrasónica aplicada y del tiempo de extracción, por lo que la aplicación de esta tecnología requiere de estudios para establecer las mejores condiciones de operación.

Herrera y Rodríguez (2016) realizaron extracción de antocianinas del agraz, utilizando método soxhlet y microondas, en los que se obtuvo 3969.7 y 3837.4 mg de cianidin-3- glucósido/100 g, respectivamente. La investigación de Puertas-Mejía, RíosYepes y Rojano (2013) trabajó sobre la extracción de antocianinas del frijol (Phaseolus vulgaris L.), utilizando el método convencional solido-líquido y extracción asistida por microondas, en los que se obtuvo 5524.18 y 5108.58 mg de cianidin-3-glucósido/100 g, respectivamente.

En el presente trabajo se obtuvo un extracto antociánico a partir del residuo de mora, con potencial aplicación como colorante aditivo para alimentos, puesto que la NTC 409 (ICONTEC, 2001) considera como colorante natural al extracto obtenido a partir de una fuente animal, vegetal o mineral; de otra parte, el extracto obtenido generaría interés para la industria alimentaria, dada la marcada tendencia hacia el consumo de productos más naturales (Parra, 2004). La extracción se realizó con etanol en solución acuosa, ya que es el mejor disolvente convencional por su disponibilidad y bajo impacto ambiental (Panić et al., 2019). Además del método soxhlet, se utilizó el proceso asistido por ultasonido para comparar el rendimiento de la extracción con esta tecnología.

En los dos métodos se varió la concentración de etanol en 75\%, 85\% y 96\% (v/v), para establecer su efecto en el rendimiento de la extracción y en la concentración de antocianinas presentes en el extracto. Finalmente, para establecer el potencial de 
recirculación del disolvente, se comparó el rendimiento y concentración de antocianinas en el extracto obtenido con etanol fresco al $96 \% \mathrm{v} / \mathrm{v}$ y etanol recuperado del rotoevaporador.

\section{Metodología}

A continuación, se describe el proceso de preparación de las muestras, la comparación de los métodos de extracción y el efecto de la concentración de la solución de etanol. Finalmente, se realiza la caracterización del extracto obtenido para establecer la cantidad de antocianinas.

\section{Acondicionamiento de la materia prima}

Se realizó la recolección del residuo generado en el proceso de despulpado de la mora de castilla y se almacenó a $2{ }^{\circ} \mathrm{C}$ en bolsas herméticas.

\section{Acidificación de la muestra}

Se preparó una solución de ácido cítrico mediante disolución de 20 g del ácido por cada $100 \mathrm{ml}$ de agua. Cada muestra de residuo se pesó, se determinó su pH y se acidificó hasta un $\mathrm{pH}$ de 2.8 .

\section{Secado de la muestra}

Se tomaron 500 g de muestra previamente acidificada y se realizó el secado a temperatura de $40{ }^{\circ} \mathrm{C}$ durante $48 \mathrm{~h}$ (Paes, Dotta y Martínez, 2013). La muestra seca se trituró en una licuadora para alimentos, CBL 999, durante 2 min para disminuir el diámetro de partícula. La muestra molida se empacó en bolsas herméticas para prevenir su oxidación.

\section{Extracción}

Los métodos de extracción utilizados fueron soxhlet y extracción asistida por ultrasonido, usando etanol como disolvente en tres concentraciones 96\%, 85\% y 75\% (v/v). Adicionalmente, después de la rotaevaporación del extracto obtenido con el etanol al $96 \%$ v/v, se realizó la extracción con el disolvente recuperado en el rotaevaporador para comparar los resultados obtenidos con el disolvente al 96\% fresco. Para cada condición experimental se realizaron tres repeticiones y se utilizó 26.6 g de muestra seca y molida.

\section{Extracción soxhlet}

La muestra se colocó en papel filtro dentro del tubo extractor y se adicionaron 400 $\mathrm{mL}$ de disolvente en un balón esmerilado. Posteriormente se realizó el calentamiento controlando la temperatura, para evitar la ebullición violenta del disolvente; por su parte, el proceso de extracción se llevó a cabo durante 240 min, aproximadamente. 


\section{Extracción asistida por ultrasonido}

La muestra se colocó en el interior de un beaker de $250 \mathrm{ml}$ y se adicionaron $400 \mathrm{ml}$ de solución extractora. Posteriormente, el beaker tapado se sumergió en un baño termostático en el interior del Ultrasonido WiseClean modelo WUC-D06H a 112.5 Watts, temperatura de $40{ }^{\circ} \mathrm{C}$ y durante $90 \mathrm{~min}$. Una vez finalizado este procedimiento, la muestra se centrifugó para separar el sólido del extracto; las condiciones de centrifugación fueron $6000 \mathrm{rpm}, 30 \mathrm{~min}$ y $10^{\circ} \mathrm{C}$.

\section{Purificación del extracto}

Se realizó la rotavaporación de la muestra centrifugada en un equipo Heidolph a temperatura de $60^{\circ} \mathrm{C}$ y presión de vacío de 175 mbar. El extracto obtenido se almacenó a $2{ }^{\circ} \mathrm{C}$ después de enfriarse en envases de color ámbar, previamente esterilizados.

\section{Caracterización cualitativa y cuantitativa de antocianinas}

Se realizó una prueba cualitativa mediante ensayo ácido-base para verificar presencia de antocianinas. La cantidad de antocianinas se determinó mediante espectrofotometría.

\section{Ensayo acido-base}

En un tubo de ensayo se adicionaron $2 \mathrm{~mL}$ de extracto y $0.8 \mathrm{~mL}$ de ácido acético glacial al $99.6 \%$; para hacer la prueba a $\mathrm{pH}$ alcalino, en otro tubo de ensayo con $2 \mathrm{~mL}$ de extracto se adicionaron $0.8 \mathrm{~mL}$ gotas de hidróxido de sodio $2 \mathrm{~N}$. Para las antocianinas en presencia de sustancias ácidas se produce una reacción de metoxilación, generando una tonalidad roja y en medios alcalinos se presenta la reacción de hidroxilación, evidenciada con el color verde (Herrera y Rodríguez, 2016).

\section{Espectrofotometría}

La cuantificación se realiza mediante el método de pH diferencial, que se basa en el cambio estructural de las antocianinas por la variación del $\mathrm{pH}$, manifestado por un cambio en la absorbancia. A pH 1 la muestra se torna coloreada porque predomina el catión flavilio, de color rojo, mientras que a pH 4.5 aparece la forma carbinol, que es incolora (Paes et al., 2013). Para el ajuste de $\mathrm{pH}$ se prepararon las soluciones buffer de cloruro de potasio $(\mathrm{KCl}) 0.025 \mathrm{M} / \mathrm{HCl} \mathrm{pH=1}$ y de acetato de sodio $\left(\mathrm{CH}_{3} \mathrm{CO}_{2} \mathrm{Na}\right)$ 0.4M/HCl pH de 4.5 (Chávez y Ordoñez, 2013).

Para la cuantificación de las antocianinas totales se empleó el método descrito por Martínez-Cruz et al. (2011), con el que se determina la absorbancia total por medio de la siguiente expresión:

$$
A=\left(A_{\lambda \max }-A_{700 \mathrm{~nm}}\right)_{p H 1}-\left(A_{\lambda \max }-A_{700 \mathrm{~nm}}\right)_{p H 4,5}
$$

El contenido total de antocianinas monoméricas y de antocianinas totales, expresadas como cianidina-3-glucósido, se calcula con las ecuaciones 2 y 3, respectivamente. 


$$
\begin{gathered}
C_{A . m .}\left(\frac{m g}{L}\right)=\frac{A \cdot P M \cdot F D \cdot 1000}{\xi \cdot L} \\
C_{A . t .}\left(\frac{m g}{L}\right)=\frac{\left(A_{\lambda \max }-A_{\lambda 700}\right)_{p H 1} \cdot P M \cdot F D \cdot 1000}{\xi * L}
\end{gathered}
$$

Dónde $C_{\text {A.m. }}$ es concentración de antocianinas monoméricas; $C_{\text {A.t. }}$, concentración de antocianinas totales; $A$, absorbancia total; $A_{\lambda \max }$, absorbancia máxima leída en el intervalo de longitud de onda (de 510 a $600 \mathrm{~nm}$ ); $P M$, la masa molecular de la cianidina3-glucósido (449.2 g/mol); FD, factor de dilución; $\xi$, absorbancia molar de la cianidina3-glucósido (26900 L/cm.mol), y L, grosor de la cubeta (1 cm).

La longitud de onda en la que se espera la máxima absorbancia $\left(A_{\lambda \max }\right)$ oscila entre 510 y 540 nm (Liu et ál., 2019; Aramwit et al., 2010), por lo cual se realizó la lectura de absorbancia para cada extracto entre 510nm y $600 \mathrm{~nm}$ y luego a $700 \mathrm{~nm}$. Cada muestra se diluyó cuarenta veces con las soluciones buffer $\mathrm{pH}=1$ y $\mathrm{pH}=4.5$; por su parte, para la lectura de absorbancias se utilizó un espectrofotómetro Jenway 6320D con precisión de $\pm 2 \mathrm{~nm}$.

\section{Cantidad de extracto}

Para los extractos de mayor concentración, se determinó el volumen y la densidad con picnómetro. El rendimiento de cada método de extracción se calculó con la ecuación 4.

$$
\% \text { rendimiento }=\frac{m_{\text {extracto }}}{m_{\text {residuo seco }}}=\frac{\rho_{\text {Ex }} \cdot V_{\text {ext }}}{m_{\text {residuo seco }}}
$$

Dónde $m_{\text {extracto }}$ es masa del extracto (g); $\rho_{E x}$, densidad del extracto $(\mathrm{g} / \mathrm{mL}) ; V_{\text {ext }}$,

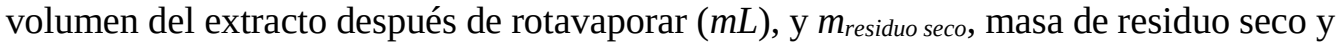
triturado (g).

\section{Resultados}

Los primeros resultados corresponden a la determinación de humedad del residuo con valor de $83.23 \pm 3.3 \%$, que coincide con lo reportado por la bibliografía, es decir, de 85\% (Ayala et al., 2013).

Como se puede observar en la Figura 1, se extrae mayor cantidad de antocianinas utilizando el método de ultrasonido con etanol al 96\% y 85\%. Como lo afirman Liu et al. (2019), a mayor concentración de etanol, hay mayor extracción, porque se favorece la presencia de etanol en la fase vapor, lo que incrementa la tasa de transferencia de masa desde el sólido hacia el disolvente. No obstante, para la extracción con etanol al 75\%, la mayor concentración de antocianinas se obtiene por el método soxhlet. En este caso, la menor concentración de etanol con ultrasonido no condujo a mayor rendimiento respecto al método soxhlet, lo cual puede atribuirse a que el tiempo y la temperatura del 
ultrasonido de mantuvieron en 90 min y $40{ }^{\circ} \mathrm{C}$. El soxhlet operó durante 240 min y a temperatura de ebullición de la solución de etanol, aproximadamente de $72{ }^{\circ} \mathrm{C}$, lo que condujo a mayor cantidad de antocianinas en el extracto. Según lo reportado por Corona-Jiménez et al. (2016), la tasa de extracción con ultrasonido depende de la combinación de las variables del proceso, es decir, tiempo, temperatura y potencia ultrasónica aplicada.

\section{Figura 1}

Rendimiento de la extracción de antocianinas.

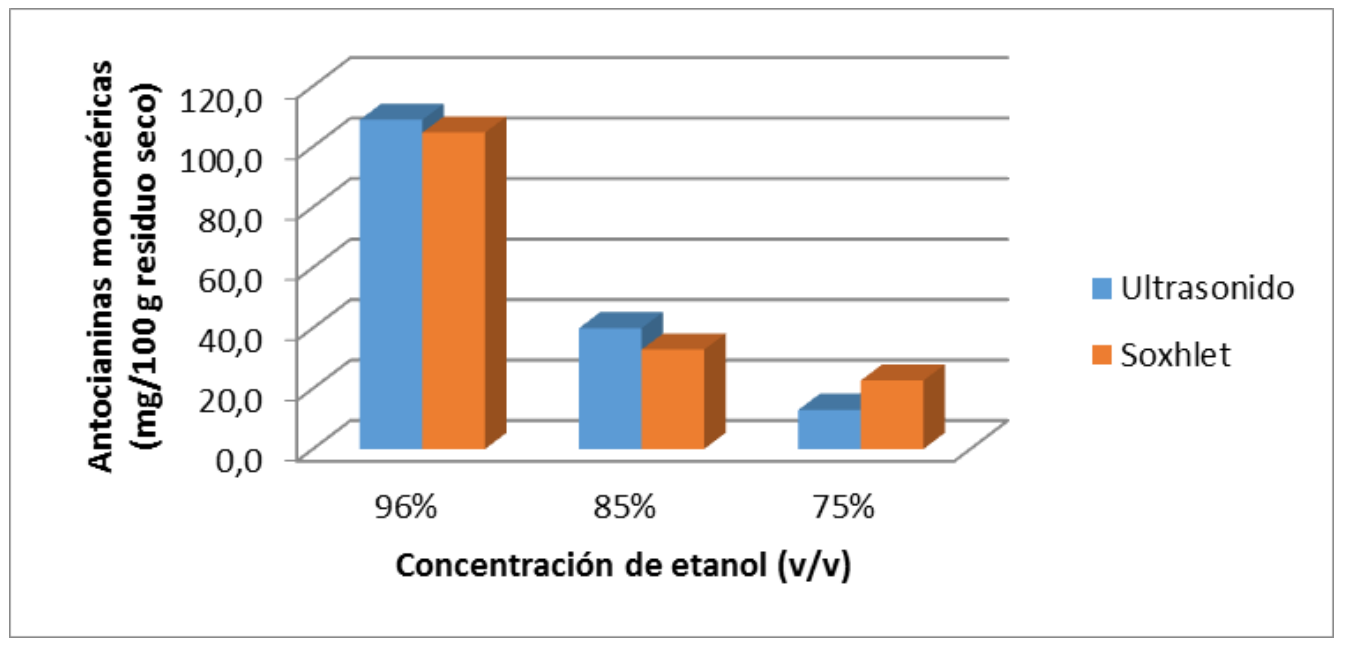

Nota. Esta es la comparación de la cantidad de antocianinas monoméricas (medidas como mg de cianidin-3-glucosido), obtenida por cada 100 g de residuo seco.

La extracción con etanol al 96\% genera la mayor concentración de antocianinas reportadas en cantidad de cianidin-3-glucósido, siendo mayoritaria por el método de ultrasonido, aunque se realizó en menor tiempo, lo que implicaría una mayor eficiencia del proceso (ver Figura 2). El mejor desempeño del ultrasonido puede asociarse a su efecto positivo en la tasa de transferencia de masa (Huang et al., 2019), aunque debería evaluarse bajo otras condiciones experimentales para validar la interacción de las variables de la operación.

Los resultados en términos de concentración son comparables con lo obtenido por Chandrasekhar et al. (2012), quienes obtuvieron $390.6 \mathrm{mg} / \mathrm{L}$ de antocianinas con etanol acidificado (50\%, v/v). En este estudio se obtuvo mayor concentración de antocianinas en la medida que se incrementó la proporción de acidulante $(\mathrm{HCl})$ en el disolvente, lo cual se atribuye a mayor estabilidad de las antocianinas a pH ácido. Por otra parte, el aumento en la concentración de etanol se presentó hasta $50 \% \mathrm{v} / \mathrm{v}$; para mayores concentraciones, según Chandrasekhar et al. (2012), se reduce la extracción de antocianinas hidrofílicas, por lo que su concentración en el extracto es menor. 
Figura 2

Concentración de antocianinas en los extractos obtenidos.

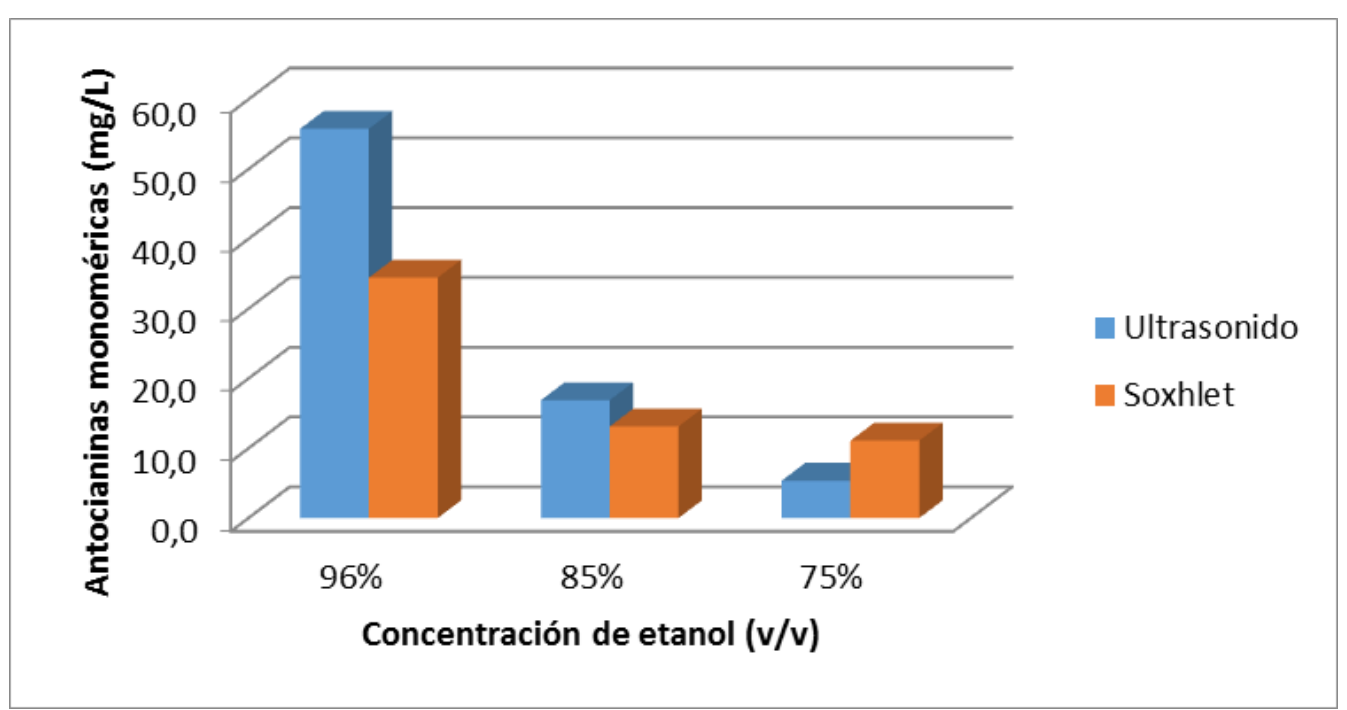

Nota. Se determinó la concentración de antocianinas monoméricas en el extracto obtenido del rotaevaporador para cada una de las condiciones experimentales evaluadas. Los extractos más concentrados también se obtuvieron con las concentraciones de etanol de 96 y $85 \%$ v/v.

Tras considerar que con los dos métodos se obtuvo el mayor rendimiento y que el extracto más concentrado fue con etanol al $96 \% \mathrm{v} / \mathrm{v}$, se procedió a realizar el proceso de extracción con el etanol recuperado en el rotaevaporador, manteniendo las mismas condiciones de tiempo, temperatura y relación disolvente/residuo seco de los ensayos originales (ver Tabla 1). El método que genera el extracto más concentrado sigue siendo el ultrasonido y con etanol $96 \% \mathrm{v} / \mathrm{v}$, generando $661.944 \mathrm{mg} / \mathrm{L}$ de antocianinas totales respecto al $465.342 \mathrm{mg} / \mathrm{L}$ producido por el método soxhlet.

\section{Tabla 1}

Efecto de utilizar etanol recuperado en concentración del 93.8\%

\begin{tabular}{|c|c|c|}
\hline $\begin{array}{c}\text { Método y concentración } \\
\text { etanol v/v }\end{array}$ & $\begin{array}{c}\text { Antocianinas totales } \\
\frac{m g \text { cianidin-3-glucósido }}{L}\end{array}$ & $\begin{array}{c}\text { Antocianinas monoméricas } \\
\frac{m g \text { cianidin-3-glucósido }}{L}\end{array}$ \\
\hline Soxhlet, etanol 96\% & $465.342 \pm 0.833$ & $34.44 \pm 0.154$ \\
\hline Soxhlet, etanol 93.8\% & $414.355 \pm 0.483$ & $29.040 \pm 0.452$ \\
\hline Ultrasonido, etanol 96\% & $661.944 \pm 1.966$ & $55.772 \pm 0.136$ \\
\hline Ultrasonido, etanol 93.8\% & $517.220 \pm 2.43$ & $40.003 \pm 0.284$ \\
\hline
\end{tabular}

Nota. La tabla muestra los resultados obtenidos para antocianinas totales y monoméricas. 
Con los dos métodos aplicados, soxhlet y ultrasonido, la concentración de antocianinas en el extracto generado con el disolvente recuperado fue menor, lo que puede deberse a la menor concentración de etanol y a la presencia de otros compuestos con el disolvente (ver Figura 3).

\section{Figura 3}

Comparación de la cantidad de extracto obtenido con etanol fresco (96\% v/v) y recuperado $(93.8 \% \mathrm{v} / \mathrm{v})$.

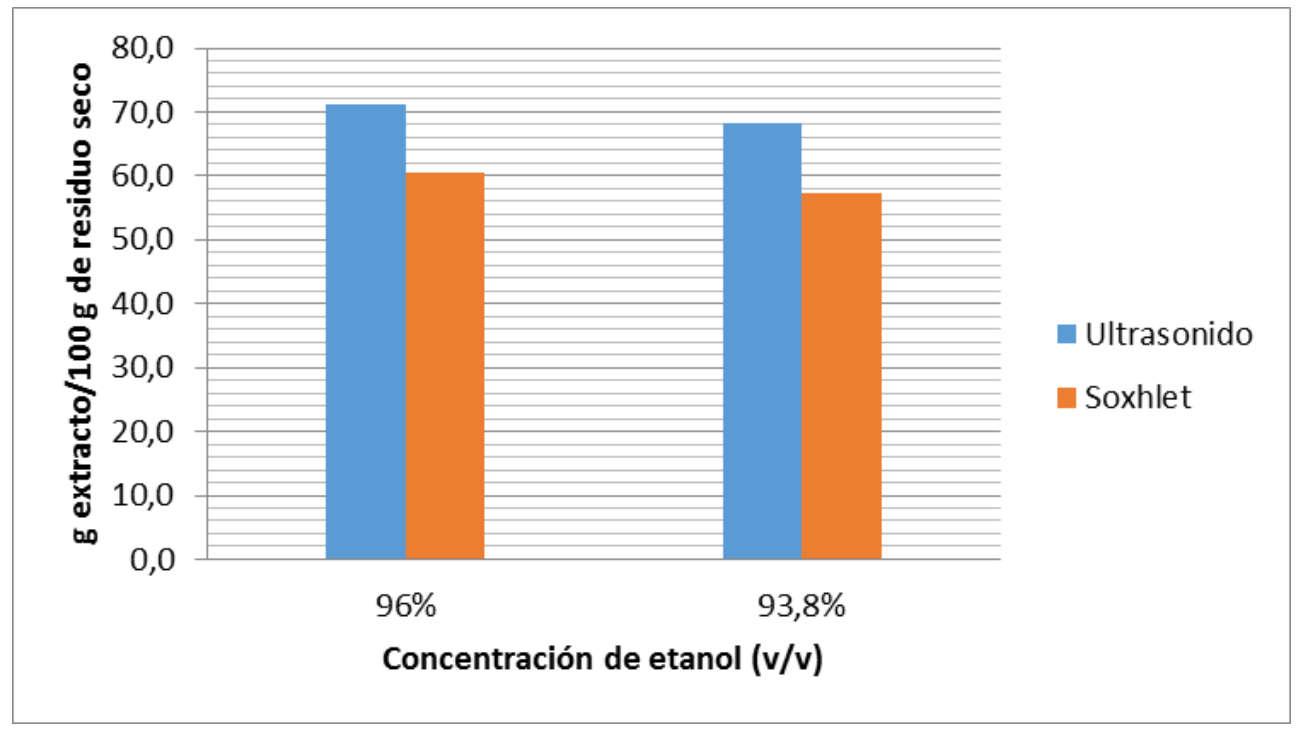

Nota. Se visualizan aquí los resultados de cantidad de extracto obtenido con los dos métodos de extracción utilizados, comparando los resultados de los extractos obtenidos con etanol al 96\% y con el etanol recuperado del rotoevaporador, que registró concentración del 93.8\%.

El rendimiento en la extracción realizada con etanol recuperado de la rotoevaporación fue inferior al obtenido con etanol fresco, lo que posiblemente se debe a la menor concentración del etanol. Heinonen et al. (2016) afirman que en la operación se extraen, en conjunto con las antocianinas, otros compuestos, como azúcares, ácidos orgánicos, proteínas, entre otros, por lo que sería necesaria la purificación del extracto, considerando que algunos de estos componentes pueden degradar las antocianinas durante el almacenamiento o dificultar el procesamiento posterior.

\section{Pruebas cualitativas}

En la Figura 4 se presentan los resultados de la prueba cualitativa aplicada a los extractos con etanol al $96 \%$ y al $85 \%$ con los dos métodos. Al variar el pH de ácido a alcalino se obtuvo coloración roja y verde, respectivamente, lo que demuestra presencia de antocianinas en los extractos obtenidos. Con esto se verifica que las antocianinas se 
comportan como indicadores ácido-base, en la medida en que varían su tonalidad según el pH (Herrera y Rodríguez, 2016).

\section{Figura 4}

Resultados del ensayo ácido-base.

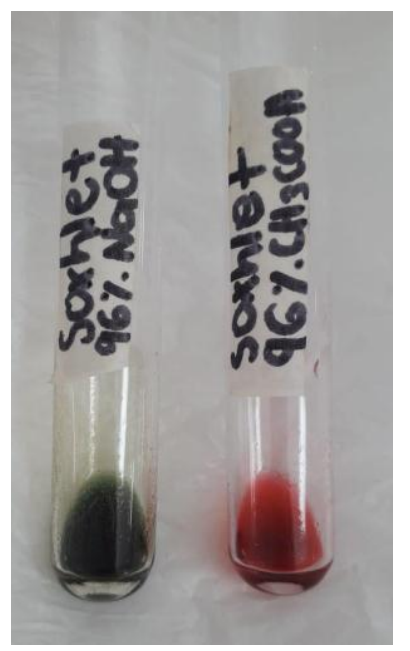

(a)

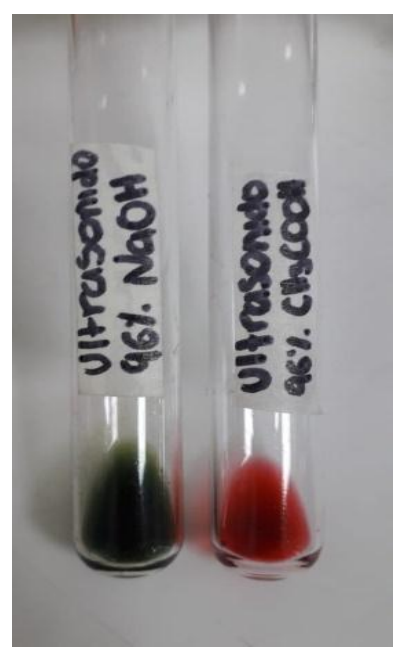

(c)

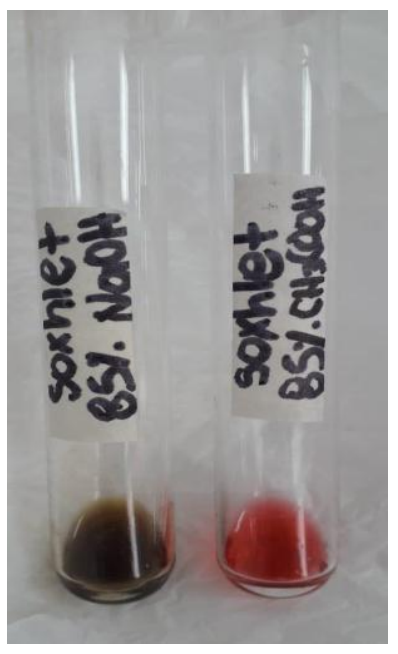

(b)

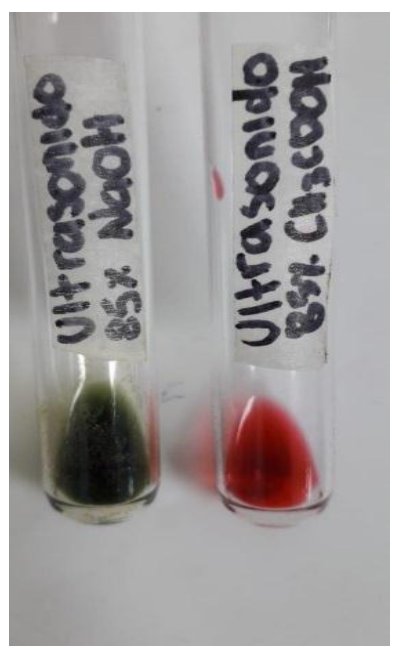

(d)

Nota. En las figuras 4a y 4b se muestran los extractos obtenidos por soxhlet a concentraciones de $96 \%$ y $85 \%$, respectivamente. Las figuras 4c y 4d evidencian los extractos obtenidos por ultrasonido y con las mismas concentraciones de etanol. 
Se comprueba de forma cualitativa la presencia de antocianinas en los extractos generados con diferentes concentraciones de etanol. Se evidencia que el mayor valor se obtiene alrededor de $540 \mathrm{~nm}$, lo que indica la presencia de antocianinas, puesto que, según Martínez-Cruz et al. (2011), en una la longitud de onda mayor a $500 \mathrm{~nm}$ se presenta la absorción máxima característica de flavonoides del grupo de las antocianinas.

\section{Figura 5}

Absorbancia en función de la longitud de onda con etanol 96\% v/v.

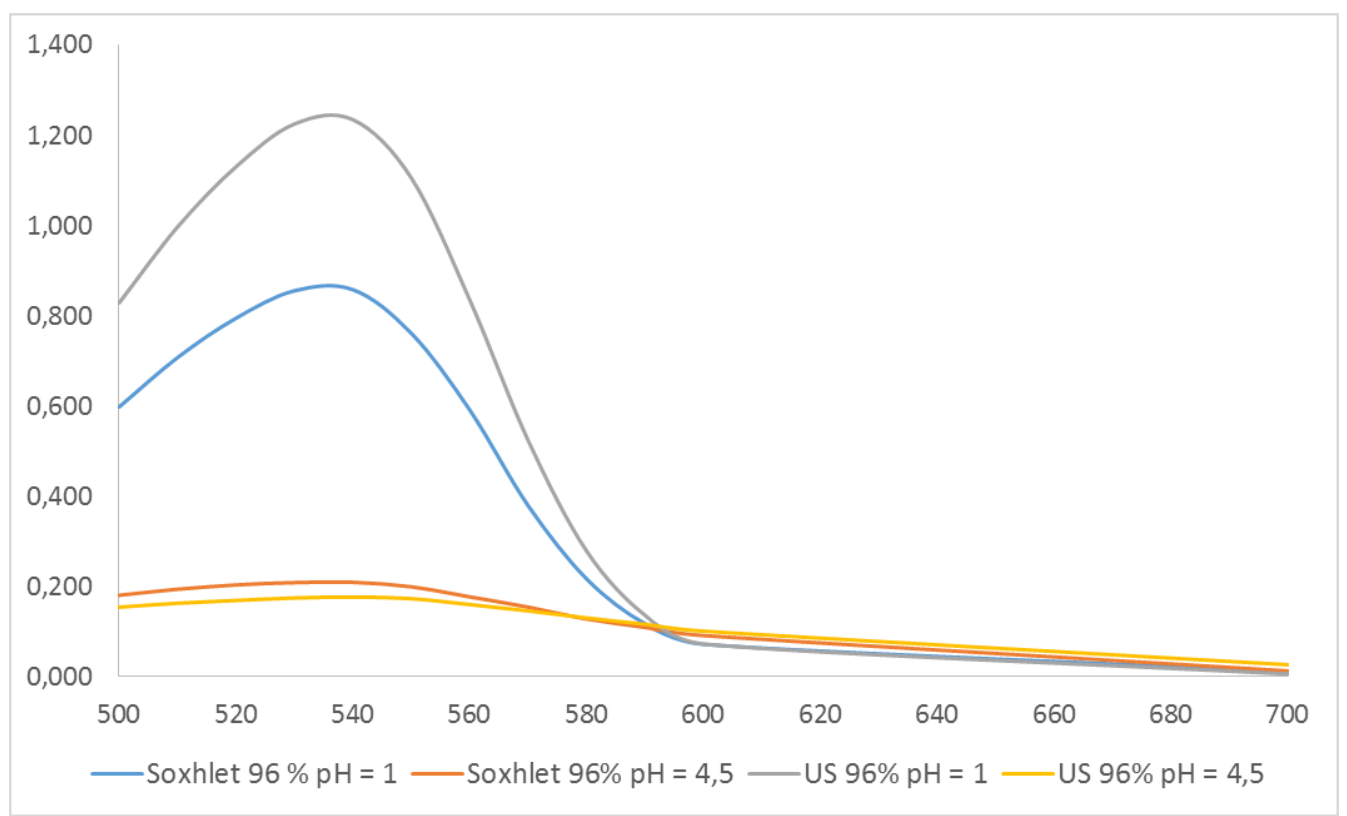

Nota. Estas son las lecturas realizadas para la absorbancia desde $510 \mathrm{~nm}$ hasta $600 \mathrm{~nm}$ y a $700 \mathrm{~nm}$.

En la Figura 5 Se verifica lo expuesto por Paes et al. (2013), porque, independiente del método soxhlet o ultrasonido, los valores más altos de absorbancia se presentan a $\mathrm{pH}$ 1.0, en el que predomina la forma coloreada de las antocianinas; por su parte, a $\mathrm{pH} 4.5$, la absorbancia es inferior a 0.2 , lo que se debe a que predomina la forma incolora.

\section{Conclusiones}

Se realizó la extracción de antocianinas con etanol, que con ultrasonido generó el mayor rendimiento medido, es decir, $71.1 \mathrm{~g}$ de extracto por $100 \mathrm{~g}$ de residuo seco. 
Adicionalmente, proporciona un extracto con $661.944 \mathrm{mg} / \mathrm{L}$ de antocianinas totales, que es la mayor concentración obtenida entre los métodos utilizados. Los mejores rendimientos y concentración en el colorante se obtuvieron con las mayores concentraciones de etanol; aunque es importante validar la interacción entre las variables de temperatura, tiempo y concentración de etanol para cada método, puesto que con etanol 75\% v/v se obtuvo mayor concentración de antocianinas con el soxhlet.

Por otra parte, se demostró que el etanol recuperado en el rotoevaporador puede ser reutilizado para posteriores extracciones, que al registrar concentración del 93.8\% evidencia una capacidad comparable con el etanol fresco. Se haría viable el proceso en la medida que se valide la cantidad de disolvente de reposición requerido dadas las pérdidas por evaporación.

Se comprobó que el extracto obtenido contiene antocianinas, lo que lo hace potencialmente aplicable como colorante natural. Al realizar el espectro de absorbancia desde $510 \mathrm{~nm}$, se verifica que la máxima absorbancia se presenta al valor esperado para el grupo funcional característico de las antocianinas. Adicionalmente, se obtuvo en el extracto el color esperado según el pH del medio.

\section{Referencias}

Aramwit, P., Bang, N., y Srichana, T. (2010). The properties and stability of anthocyanins in mulberry fruits. Food Research International, 43(4),10931097. doi: 10.1016/j.foodres.2010.01.022

Ayala, L., Valenzuela, C., y Bohórquez, Y. (2013). Caracterización fisicoquímica de mora de castilla (Rubus glaucus Benth) en seis estados de madurez. Biotecnología en el Sector Agropecuario y Agroindustria, 11(2), 10-18.

Cai, Z., Qu, Z., Lan, Y., Zhao, S., Ma, X., Wan, Q., Jing, P., y Li, P. (2016). Conventional, ultrasound-assisted, and accelerated-solvent extractions of anthocyanins from purple sweet potatoes. Food Chemistry, 197(part. A), 266272. doi: 10.1016/j.foodchem.2015.10.110

Chandrasekhar, J., Madhusudhan, M., y Raghavarao, K. (2012). Extraction of anthocyanins from red cabbage and purification using adsorption. Food and Bioproducts Processing, 90(4), 615-623. doi: 10.1016/j.fbp.2012.07.004

Chávez, R., y Ordoñez, E. (2013). Polifenoles totales, antocianinas y capacidad antioxidante (DPPH y ABTS) durante el procesamiento del licor y polvo de cacao. Revista ECIPerú, 10(1), 42-52.

Corona-Jiménez, E., Martínez Navarrete, N., Ruiz-Espinoza, H., y Carranza-Concha, J. (2016). Extracción asistida por ultrasonido de compuestos fenólicos de semillas de chia (Salvia hispanica L.) y su actividad antioxidante. Agrociencia, 50(4), 403-412. 
Garzón, G. (2008). Las antocianinas como colorantes naturales y compuestos bioactivos: revisión. Acta Biológica Colombiana, 13(3), 27-36.

Guo, N., Ping-Kou, Jiang, Y.-W., Wang, L.-T., Niu, L.-J., Liu, Z.-M., Fu, Y.-J (2019). Natural deep eutectic solvents couple with integrative extraction technique as an effective approach for mulberry anthocyanin extraction. Food Chemistry, 296, 78-85. doi: 10.1016/j.foodchem.2019.05.196

Heinonen, J., Farahmandazad, H., Vuorinen, A., Kallio, H., Yang, B., y Sainio, T. (2016). Extraction and purification of anthocyanins from purple-fleshed potato. Food and Bioproducts Processing, 99,136-146. doi: 10.1016/j.fbp.2016.05.004

Herrera, X., y Rodríguez, K. (2016). Evaluación del extracto de flavonoles y antocianinas contenidos en el agraz (Vaccinium Meridionale Swartz) obtenidos a nivel laboratorio por medio de los métodos de extracción por solventes y extracción asistida por microondas. Fundación Universidad de América.

Huang, H., Belwal, T., Aalim, H., Li, L., Lin, X., Liu, S., Ma, C., Li, Q., Zou, Y., Luo, Z. (2019). Ultrasonic impact on viscosity and extraction efficiency of polyethylene glycol: a greener approach for anthocyanins recovery from purple sweet potato. Food Chemistry, 283, 59-67. doi: 10.1016/j.foodchem.2019.01.017

Icontec. (2001). NTC 409. Industrias alimentarias. Colorantes aditivos para alimentos. Icontec. Recuperado de: http://files.aditalimentarios.webnode.es/200000042ea1dcec11e/NTC409_colorantes\%20aditivos\%20para\%20alimentos.pdf

Khalifa, I., Zhu, W., Li, K., y Li, C. (2018). Polyphenols of mulberry fruits as multifaceted compounds: compositions, metabolism, health benefits, and stability—a structural review. Journal of Functional Foods, 40, 28-43. doi: 10.1016/j.jff.2017.10.041

Liu, W., Yang, C., Zhou, C., Wen, Z., y Dong, X. (2019). An improved microwaveassisted extraction of anthocyanins from purple sweet potato in favor of subsequent comprehensive utilization of pomace. Food and Bioproducts Processing, 115, 1-9. doi: 10.1016/j.fbp.2019.02.003

Martínez-Cruz, N., Arévalo-Niño, K., Verde-Star M., M., Rivas-Morales, C., OrandayCárdenas, A., Núñez-González, M., y Morales-Rubio, M. (2011). Antocianinas y actividad antiradicales libres de Rubus adenotrichus Schltdl (zarzamora). Revista Mexicana de Ciencias Farmacéuticas, 42(4), 66-71.

Ministerio de Agricultura y Desarrollo Rural y Dane. (2013). El cultivo de la mora de Castilla (Rubus glucus Benth) frutal de clima frío moderado, con propiedades curativas para la salud humana. Boletín Mensual Insumos y Factores Asociados a la producción agropecuaria, 17, 1-6. 
Paes, J., Dotta, R., y Martínez, J. (2013). Estraction of phenolic compounds from blueberry (Vaccinium myrtillus L.) residues using supercritical CO2 and pressurized water. En III Iberoamerican Conference on Supercritical Fluids. Conferencia presentada en Cartagena de Indias, Colombia. doi: 10.1016/j.supflu.2014.07.025

Panić, M., Gunjević, V., Cravotto, G., y Radojčić Redovniković, I. (2019). Enabling technologies for the extraction of grape-pomace anthocyanins using natural deep eutectic solvents in up-to-half-litre batches extraction of grape-pomace anthocyanins using NADES. Food Chemistry, 300, 125185. doi: 10.1016/j.foodchem.2019.125185

Parra, V. (2004). Estudio comparativo en el uso de colorantes naturales y sintéticos en alimentos, desde el punto de vista funcional y toxicológico (tesis de pregrado). Facultad de Ciencias Agrarias, Universidad Austral de Chile.

Puertas-Mejía, M., Ríos-Yepes, Y., y Rojano, B. (2013). Determinación de antocianinas mediante extracción asistida por radiación de microondas en frijol (Phaseolus vulgaris L.) de alto consumo en Antioquia-Colombia. Revista Cubana de Plantas Medicinales, 18(2), 288-297.

Yepes, S., Montoya, L., y Orozco, F. (2008). Valorización de residuos agroindustriales - frutas -en Medellín y el sur del Valle de Aburrá, Colombia. Revista Facultad Nacional de Agronomía, 61(1), 4422-4431. 